\title{
Liver Tumor Biopsy in a Respiring Phantom with the Assistance of a Novel Electromagnetic Navigation Device
}

\author{
Filip Banovac ${ }^{1,2}$, Neil Glossop ${ }^{3}$, David Lindisch ${ }^{1}$, \\ Daigo Tanaka $^{1}$, Elliot Levy ${ }^{1,2}$, and Kevin Cleary ${ }^{1}$ \\ ${ }^{1}$ Imaging Sciences and Information Systems Center (ISIS), Department of Radiology, \\ Georgetown University, 2115 Wisconsin Avenue, Suite 603, Washington, DC, U.S.A. \\ \{Banovac, Lindisch, Tanaka, Levy, Cleary\} @isis.imac.georgetown. edu \\ ${ }^{2}$ Georgetown University Hospital, 3800 Reservoir Road, N.W., Washington, DC, U.S.A. \\ ${ }^{3}$ Traxtal Technologies LLC, 5116 Bissonnet, Bellaire, TX 77401
}

\begin{abstract}
The purpose of this study was to evaluate our ability to insert magnetically tracked needles into liver phantom tumors which move simulating physiologic respiration. First, a novel image-guided platform based on a new magnetic tracking device (AURORA ${ }^{\mathrm{TM}}$ ) was constructed. Second, an accuracy evaluation of a compatible magnetically tracked needle (MagTrax) was performed. Finally, 16 liver tumor punctures were attempted using only the image-guided platform for guidance. The inherent MagTrax needle positional error was $0.71 \pm 0.43 \mathrm{~mm}$ in the non-surgical laboratory setting. Successful puncture of liver tumors was achieved in 14 of 16 attempts $(87.5 \%)$ by two us-

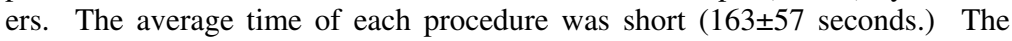
system adequately displayed the moving liver allowing for tumor target visualization and targeting. The AURORA based navigation platform and the compatible MagTrax needle appear promising for more rigorous phantom accuracy studies and in vivo tumor puncture testing in a respiring animal.
\end{abstract}

\section{Introduction}

Image-guided systems for intervention in the thorax and abdomen have not been developed, in part because of problems related to organ motion induced by respiration. The internal organs are not rigid nor directly accessible and therefore difficult to track and register for purposes of image guidance. This is in contrast to intracranial and musculoskeletal interventions where image-guided systems based on bony landmarks have been developed by many researchers and commercial systems are available.

In particular, the need for organ tracking and precision instrument placement in liver procedures has multiple clinical justifications. Tumor biopsy, radiofrequency ablation of tumors, portal and hepatic venous access for intrahepatic shunts, and billiary access for drainage all require precision for procedural success. The liver predominately moves in a cranio-caudal direction during quiescent physiologic breathing exhibiting displacements from 10 to $25 \mathrm{~mm}[1,2]$. For open surgery, Herline et al. explored the feasibility of surface based registration methods for intraoperative liver tracking [3]. They also showed the feasibility of liver tracking in open and laparoscopic procedures [4]. However, for percutaneous minimally invasive procedures, the only clinically accepted methods are direct visualization with fluoroscopy or ultrasound, each of which has its own shortcomings. 
Several image-guided surgical systems based on magnetic position tracking are currently commercially available. BioSense Webster, a Johnson and Johnson company, offers two navigational systems for cardiac catheterization and mapping, the NOGA $^{\mathrm{TM}}$ and CARTO ${ }^{\mathrm{TM}}$ systems. This product has been used in early clinical studies showing feasibility for intracranial neuro-navigation [5] and cardiac mapping in treatment of arrhythmias [6]. Solomon et al. used the Biosense system to assist in placement of a transjugular intrahepatic portosystemic shunt (TIPS) in swine [7]. For endoscopic sinus surgery, Visualization Technologies Inc. a subsidiary of General Electric (Lawrence, MA) sells the InstaTrack 3000® image-guided surgery system.

A magnetic positioning guidance system that is targeted at intra-abdominal interventions is the UltraGuide1000 (UltraGuide, Tirat Hacarmel, Israel). The UltraGuide device was introduced to complement currently used sonographic guidance techniques, especially to enhance the freehand techniques. The device uses small magnetic sensors attached to the hub or the shaft of the needle to help the user navigate the needle to the target. Howard et al. and Krombach et al. independently reported the successful use of UltraGuide to perform liver and kidney percutaneous procedures respectively $[8,9]$. Wood et al. reported the use of the same device in RF ablation of renal cell carcinoma [10].

The purpose of this study was to evaluate the usefulness of magnetic tracking and image guidance for precision biopsy of simulated lesions in a moving liver phantom. This study was based on a liver respiratory motion simulator developed by our group and the AURORA magnetic tracking system under development by Northern Digital Inc., Ontario, Canada. An accuracy evaluation of a newly developed, commercially available and AURORA compatible needle was also performed.

\section{Materials and Methods}

\subsection{Liver Respiratory Motion Simulator}

To evaluate magnetic tracking for minimally invasive abdominal interventions, the Georgetown group has developed a liver respiratory motion simulator. The simulator includes a synthetic liver mounted on a motion platform. The simulator consists of a dummy torso, a synthetic liver model, a motion platform, a graphical user interface, the AURORA magnetic tracking system, and a magnetically tracked needle and catheter as previously described $[11,12]$.

\subsection{Liver Phantom}

A human torso model containing a liver phantom was modified from our previously described prototype [12]. The liver phantom was made from a two part flexible foam (FlexFoam III, Smooth-On, Easton, PA) which was cast from a custom made mold. The foam material was cured to approximately simulate liver tissue resistance to needle puncture. Two spiculated, silicone, elliptical tumors (maximum diameters of 3.1 and $2.2 \mathrm{~cm}$ ) containing radio-opaque CT contrast were incorporated into the liver model prior to curing to serve as tumor targets. The liver was attached to a linear motion platform at the base of the torso's right abdomen (Figure 1). 
The platform can be programmed to simulate physiologic cranio-caudal motion of the liver with options for respiratory rate control, breath depth, and breath pause (breath hold). A ribcage and single layer latex skin material (Limbs and Things, Bristol, UK) were added for aesthetic and physical reality.

\subsection{Magnetic Tracking Device and Sensors}

A prototype of a new magnetic field based tracking system, the AURORA,

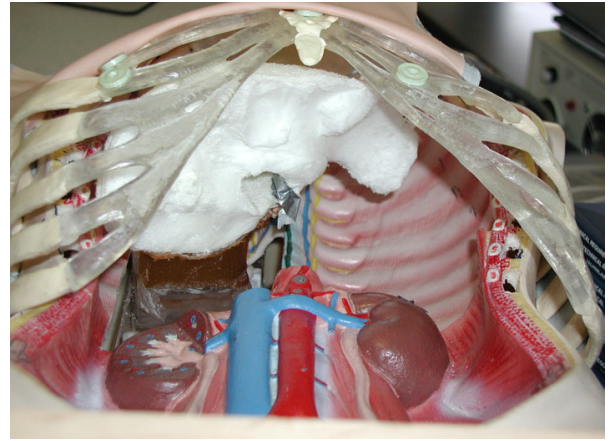

Fig. 1. A foam liver model (white) mounted on a linear platform inside the torso model was used in the experiments. The system consists of a control unit, sensor interface device, and field generator as shown in Figure 2.

The AURORA uses cylindrically shaped sensors that are extremely small $(0.9 \mathrm{~mm}$ in diameter and $8 \mathrm{~mm}$ in length). This enables the sensors to be embedded into surgical instruments. We used two magnetically tracked instruments in these experiments: 1) A prototype 5-French catheter with an embedded sensor coil was provided by the manufacturer; and 2) A needle/probe combination (MagTrax) as shown in Figure 3.

The MagTrax (Traxtal Technologies, Houston, Texas) needle/probe consists of a $15 \mathrm{~cm}$ stylette with a magnetic sensor at its tip and an 18-gauge trocar. This instrument was used in the study to puncture the tumors.

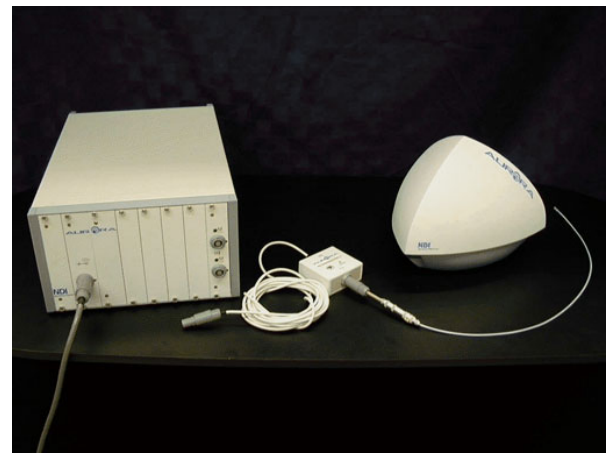

Fig. 2. AURORA control unit and field generator (courtesy of Northern Digital Inc.)

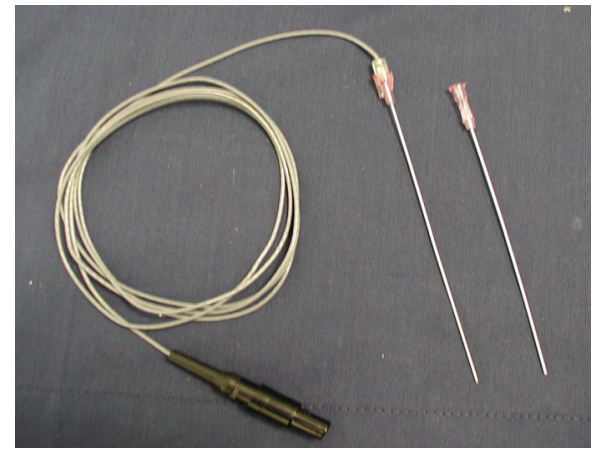

Fig. 3. MagTrax needle/probe with a stylette containing a magnetic sensor in its tip and leads exiting the hub. An 18-gauge trocar is seen on the right.

\subsection{Guidance System and Software}

A PC-based software application called ROGS (Respiring Organ Guidance System) was developed to assist the physician in performing the puncture of the liver parenchyma and needle guidance into the liver tumors. The system incorporates a graphi- 
cal user interface [13] shown in Figure 4. The ROGS software allows for the loading of serial axial CT images, pre-procedural planning to the target of interest, tracking of respiratory motion, and real-time display of the biopsy needle as it approaches the target tumor. The sequence of steps in path planning and needle placement is shown in Figure 5.

\subsection{MagTrax Needle/Probe Accuracy Evaluation}

A MagTrax needle/probe containing a single five degree of freedom magnetically tracked sensor was solidly fixed to two passive optically tracked rigid bodies (small $50 \times 50 \mathrm{~mm}$ and large $95 \times 95 \mathrm{~mm}$ ). The sensor assembly was moved randomly through 101 positions in a volume of $36 \mathrm{~mm}$ x $36 \mathrm{~mm}$ x $47 \mathrm{~mm}$. At each location the sensor assembly was clamped and 10 samples from each of the targets were collected by the POLARIS optical system (Northern Digital Inc, Ontario, Canada) and AURORA magnetic system. The data sets were aligned by mathematical transformations and the difference in position and orientation of the two POLARIS sensors (control) versus the larger POLARIS sensor and MagTrax probe were calculated over the 101 positions. The experiment was performed in the absence of ferromagnetic interference.

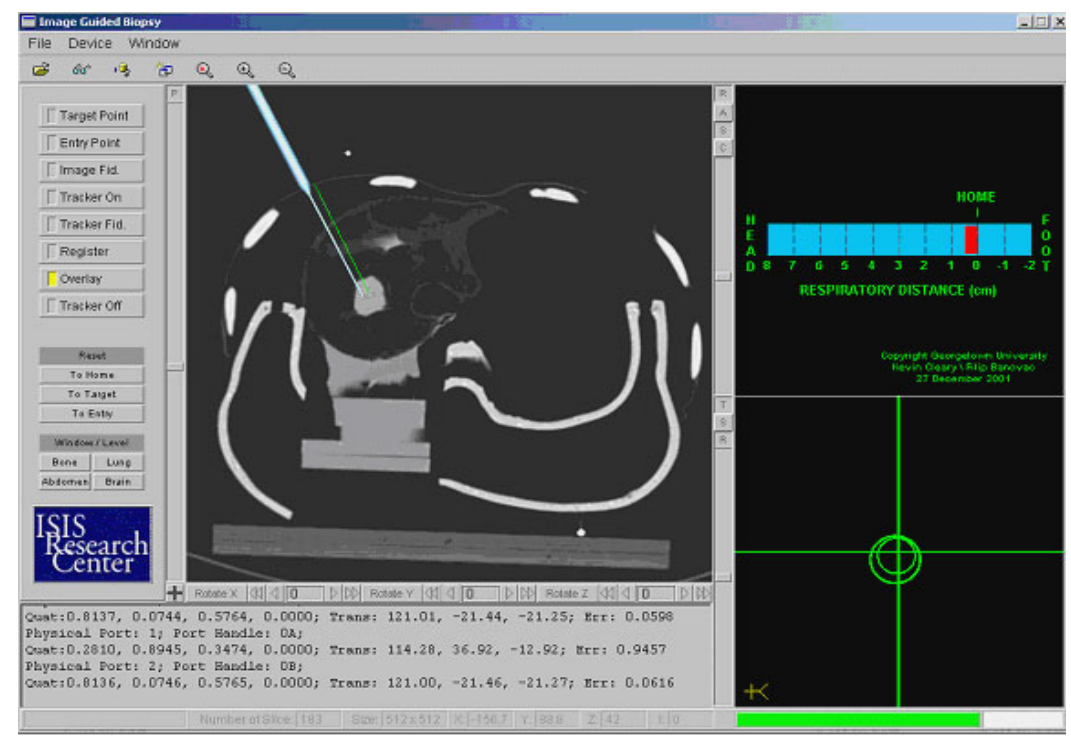

Fig. 4. Graphical user interface (center window shows probe overlaid on image, respiratory tracking in upper right, targeting window in lower right, patent pending 2001-2002)

\subsection{Real-Time Tumor Biopsy Evaluation}

A series of tumor targeting experiments were performed to test the usefulness of the system in accurately guiding a user to a target while the phantom resumes physiologic respiration. Two users (F.B. and D.L) independently performed 8 punctures each. The experimental design was divided into three stages as follows: 

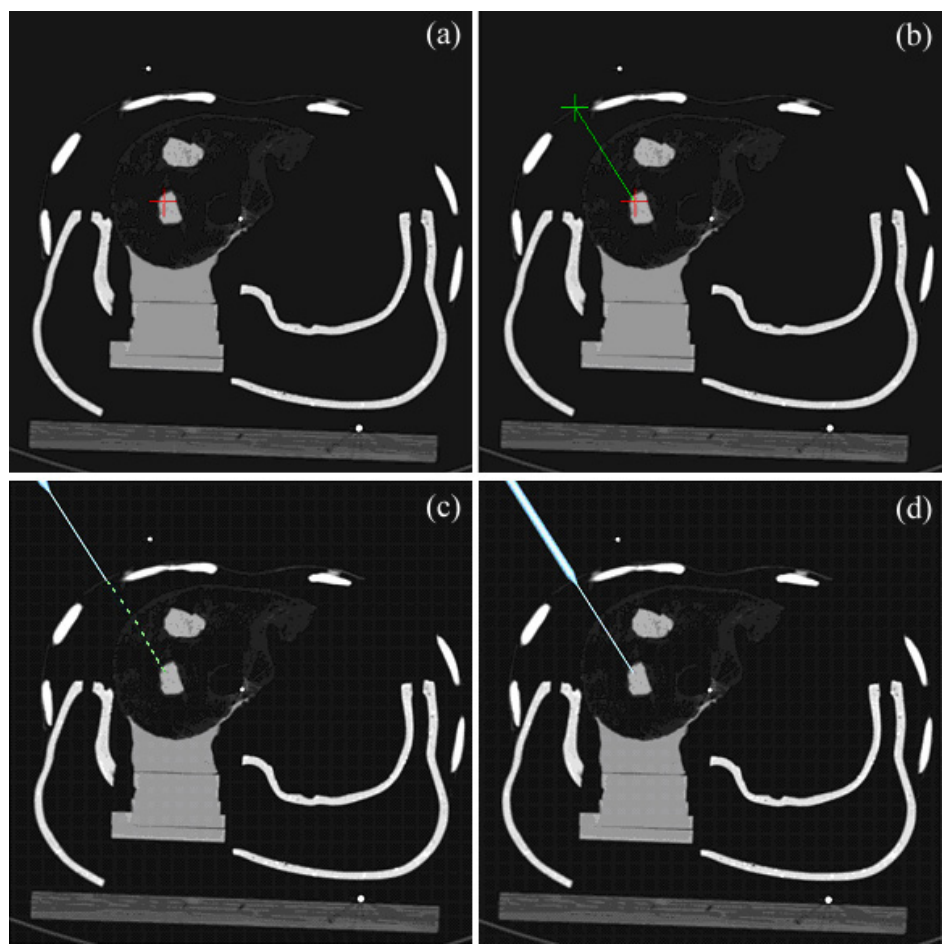

Fig. 5. Target tumor on an axial image of the phantom torso is selected by the radiologist (a); subsequently, the radiologist selects the skin entry point (b) and a planned path appears on the reconstructed image; the needle/probe is placed at the skin entry point (c) using cross hairs targeting window (Fig 4. lower right); finally, the needle is driven into the tumor (d) along the planned path indicated by the dotted line

\section{Stage 1: CT Scanning and Registration}

1. A magnetically tracked catheter was wedged in the hepatic vein of the liver. Several skin fiducials (multimodality markers, IZI Medical, Baltimore, Maryland) were placed on the rib cage.

2. A series of $3 \mathrm{~mm}$ axial slices with $1 \mathrm{~mm}$ axial reconstructions were obtained on CT VolumeZoom (Siemens, Erlangen, Germany) from the base of the lungs through the liver while the liver was kept in end-inspiration (simulating the breath-hold technique used in clinical practice).

3. The images were transferred to the ROGS using the DICOM standard.

4. The tracking catheter was left in the hepatic vein and the simulator was moved to the interventional radiology suite. The magnetic field generator was positioned near the phantom above the chest.

5. The position of the wedged catheter was read in the magnetic coordinate system. The position of the skin fiducials were read in the magnetic coordinate system by touching each fiducial with the MagTrax needle.

6. The position of the catheter and fiducials was determined in CT coordinate space by prompting the user to select these same points on the CT images. 
7. A least-squares fit registration algorithm was invoked to determine the transformation matrix from magnetic space to CT space.

\section{Stage 2: Biopsy Path Planning Phase}

8. Each user was allowed one practice "planning phase" and "puncture (biopsy) phase" to get familiarized with the ROGS.

9. The user selected the target and a suitable skin entry point by scrolling through the axial images (figure $5 \mathrm{a}$ and $5 \mathrm{~b}$ ) thus selecting a biopsy path.

10. Simulated respirations were initiated at 12 breaths per minute with $2 \mathrm{~cm}$ cranio-caudal liver excursion.

\section{Stage 3: Biopsy Phase}

11. The MagTrax needle/probe was positioned on the skin entry point as determined in the "planning phase" and displayed by the ROGS overlay.

12. A real-time display of the current liver position was displayed by the ROGS system based on the position of the magnetically tracked catheter.

13. The MagTrax needle was tracked in real-time and the transformation matrix computed in step 7 was used to compute the overlay of the probe on the CT images which were reconstructed so to show the planned path of the needle.

14. When satisfied with the target position relative to the planned path, the user would initiate temporary cessation of respiration (simulating a 20 second breath hold in clinical practice). If the allotted time was exceeded, the phantom would continue spontaneous respirations for a minimum of $20 \mathrm{sec}-$ onds (hyperventilation in clinical practice). Any partially inserted needle would be left in place as is frequently done during biopsy procedures.

15. Repeating step 14 the user would keep making minor adjustments to the needle until satisfied with the needle position as displayed on ROGS.

16. The time for each "planning phase" and "biopsy phase" were recorded. Multi-projection fluoroscopic images were taken at the end of each needle placement to ascertain whether the target tumor was successfully punctured.

\section{Results}

\subsection{Accuracy Evaluation of the MagTrax Needle}

Using the optical passive tracking system as the gold standard as described in the methods in Section 2.5, the mean measurement error and standard deviation of the MagTrax needle/probe using the AURORA system was $0.71 \pm 0.43 \mathrm{~mm}(\mathrm{n}=101)$ in a non-surgical environment. The maximum error noted was $2.96 \mathrm{~mm}$.

\subsection{Tumor Biopsy Evaluation}

The targeted tumor was successfully punctured in 14 out of 16 biopsy attempts $(87.5 \%)$. This was done without any additional real-time imaging guidance such as fluoroscopy. Instead, fluoroscopy was used to confirm the final location of the needle and evaluate accuracy.

Each user missed the target tumor once. In those instances, the maximal tangential distance from the lesion to the needle was $3.98 \mathrm{~mm}$. On most occasions, the user was 
able to reach the tumor in a single continuous puncture after the needle was positioned on the skin entry point. This was done within a single 20 second breath hold (pause in liver motion) in end-inspiratory liver position. More than two breath hold cycles with intervening period of hyperventilation were needed on only 1 out of 16 experimental trials. The time needed for registration ranged from 173-254 seconds. The planning time, needle manipulation time, and total procedure times for the 16 trials are presented in Table 1.

Table 1. Planning, needle manipulation and total procedure times for ROGS assisted biopsy of tumors in a respiring liver phantom

\begin{tabular}{|l|c|c|c|}
\hline & $\begin{array}{c}\text { Mean Planning } \\
\text { Time (s) } \pm \text { SD }\end{array}$ & $\begin{array}{c}\text { Needle Manipulation } \\
\text { Biopsy Time (s) } \pm \text { SD }\end{array}$ & $\begin{array}{c}\text { Total Procedure } \\
\text { Time (s) } \pm \text { SD }\end{array}$ \\
\hline User 1 & $72 \pm 35$ & $79 \pm 40$ & $151 \pm 59$ \\
User 2 & $61 \pm 31$ & $111 \pm 41$ & $172 \pm 43$ \\
Overall & $71 \pm 36$ & $93 \pm 43$ & $163 \pm 57$ \\
\hline
\end{tabular}

\section{Discussion}

Image-guided surgery is now an established practice for brain, ENT, and spinal procedures. These systems are based on optical tracking and bony landmarks. The introduction of a new magnetic tracking system with sensor coils small enough to be embedded into instruments may enable the development of image-guidance for abdominal and thoracic internal organs.

The overall goal of the research described here is to develop magnetic tracking for internal organs, including methods of compensating for respiratory motion. The initial results presented here show the feasibility of magnetic tracking, but much work remains to be done before this technology can implemented in clinical practice.

The accuracy of the MagTrax needle/probe used with the AURORA was measured as $0.71 \mathrm{~mm}$. This should be sufficient for clinical practice. Additionally, the location of the magnetic sensor in the tip of the needle/probe means the instrument is not subject to errors introduced by needle bending unlike those used in the UltraGuide system where the proximal end of the needle is tracked [8].

The ROGS interface allowed a high success rate $(87.5 \%)$ for needle puncture of the two small to medium sized simulated tumors. Most notably, the procedure was done while actively tracking the physiologic motion of the liver. To our knowledge, ROGS is the first system that allows real-time compensation for the moving intraabdominal target and subsequent compensated guidance for the needle puncture. The system was easy to use requiring only a single practice attempt to attain a satisfactory comfort level. The entire average procedure time lasted less than three minutes which is shorter than the time needed to perform this task during a conventional CT guided biopsy. These initial results are promising towards the development of a clinically useful system. Further experiments and animal studies are planned. 


\section{Acknowledgements}

This work was funded by U.S. Army grant DAMD17-99-1-9022, an NIH National Research Service Award Fellowship (F32HL68394-01), and a CIRREF Academic Transition Award.. The content of this manuscript does not necessarily reflect the position or policy of the U.S. Government. The authors would like to thank Northern Digital, Inc., for the loan of the magnetic tracking system. The software was developed by Daigo Tanaka of Georgetown University and Sheng Xu, a graduate student in the NSF-funded Center for Computer Integrated Surgical Systems and Technologies at Johns Hopkins University.

\section{References}

1. Davies, S.C., et al., Ultrasound quantitation of respiratory organ motion in the upper abdomen. Br J Radiol, 1994. 67(803): p. 1096-102.

2. Suramo, I., M. Paivansalo, and V. Myllyla, Cranio-caudal movements of the liver, pancreas and kidneys in respiration. Acta Radiol Diagn (Stockh), 1984. 25(2): p. 129-31.

3. Herline, A.J., et al., Surface registration for use in interactive, image-guided liver surgery. Comput Aided Surg, 2000. 5(1): p. 11-7.

4. Herline, A.J., et al., Image-guided surgery: preliminary feasibility studies of frameless stereotactic liver surgery. Arch Surg, 1999. 134(6): p. 644-9; discussion 649-50.

5. Zaaroor, M., et al., Novel magnetic technology for intraoperative intracranial frameless navigation: in vivo and in vitro results. Neurosurgery, 2001. 48(5): p. 1100-7

6. Gepstein, L., G. Hayam, and S.A. Ben-Haim, A novel method for nonfluoroscopic catheterbased electroanatomical mapping of the heart. In vitro and in vivo accuracy results. Circulation, 1997. 95(6): p. 1611-22.

7. Solomon, S.B., et al., TIPS placement in swine, guided by electromagnetic real-time needle tip localization displayed on previously acquired 3-D CT. Cardiovasc Intervent Radiol, 1999. 22(5): p. 411-4.

8. Howard, M.H., et al., An electronic device for needle placement during sonographically guided percutaneous intervention. Radiology, 2001. 218(3): p. 905-11.

9. Krombach, G.A., et al., US-guided nephrostomy with the aid of a magnetic field-based navigation device in the porcine pelvicaliceal system. J Vasc Interv Radiol, 2001. 12(5): p. 623-8.

10. Wood, B.J., et al., Percutaneous radiofrequency ablation with three-dimensional position sensor guidance. Cardiovasc Intervent Radiol, 2001(in print).

11. Cleary, K., et al. Development of a Liver Respiratory Motion Simulator to Investigate Magnetic Tracking for Abdominal Interventions. in SPIE Medical Imaging. 2002. San Diego, CA.

12. Banovac, F., et al. Design and Construction of a Liver Phantom for CT Imaging and Interventions that Simulates Liver Motion Seen During Respiration. in Radiologic Society of North America. 2001. Chicago, IL.

13. Cleary, K., et al. Feasibility of Magnetic Tracking for Image-Guided Abdominal Interventions Based on a Liver Respiratory Motion Simulator. submitted to IEEE International Symposium on Biomedical Imaging. 2002. Washington, DC. 